\title{
Ground-dwelling arthropod (Araneae, Coleoptera: Carabidae, Isopoda: Oniscidea) assemblages on Hungarian main road verges
}

\author{
Diána VONA-TÚRI, ${ }^{1}$ Tünde SZMATONA-TÚRI, ${ }^{2}$ Ferenc KÁDÁR, ${ }^{3}$ \\ Balázs KISS, ${ }^{3}$ András WEIPERTH,${ }^{4}$ Blanka GÁL ${ }^{4}$ \\ ${ }^{1}$ Eötvös József Reformed Education Center, H-3360 Heves, 29. Dobó Street, Hungary, \\ e-mail: turidiana79@gmail.com (*corresponding author) \\ ${ }^{2}$ Forestry, Agricultural and Game Management Training School and Student Hostel of Mátra, \\ H-3232 Mátrafüred, 11. Erdész Street, Hungary, \\ e-mail: turitunde79@gmail.com \\ ${ }^{3}$ Centre for Agricurtural Research, Hungarian Academy of Sciences, Plain Protection Institute, \\ H1022 Budapest, Herman Ottó út 15. \\ e-mail:kadar.ferenc@agrar.mta.hu,kiss.balazs@agrar.mta.hu \\ ${ }^{4}$ Centre for Ecological Research, Hungarian Academy of Sciences, Danube Research Institute, \\ H 1113 Budapest, Karolin út 29. \\ e-mail: weiperth.andras@okologia.mta.hu,gal.blanka@okologia.mta.hu
}

Manuscript received Sept. 30, 2016; revised Oct. 20, 2016; Accepted Oct. 22, 2016

\begin{abstract}
In this study, we research ground-dwelling arthropods along four road stretches, which represent the main verge habitats (arid grassland, agricultural area, forest, and wetland) of Hungary. Besides the faunistic investigation of arthropod communities, we described and compared dominance relation and species composition. We identified 83 carabids, 81 spiders, and 4 isopod species. Seven beetle species and one spider species are included in the invertebrate Red List of Hungary. The registered species were dominant on main road verges bordering arid grassland and wetland. Main road verges proved to be a diverse habitat; hence, it is important to carry on more investigation.
\end{abstract}

Keywords: spider, beetle, woodlice, species richness, abundance, diversity

\section{Introduction}

The establishment of road networks is one of the most prevalent ways of land usage these days with more and more studies focusing on the effect of roads on 
biodiversity [1]. Road ecology is a new discipline nowadays [2]. Researchers of roadside and highway verges provide several data about beetles $[3,4,5,6,7,8,9]$, spiders [3, 6, 7, 10], and isopods [11, 12, 13, 14]. Trombulak and Frissell [15] examined the short- and long-term effects of roads on environment. The studies of Holderegger and Di Giulio [16] targeted the effects of barriers on mortality and gene replacement; also, Forman et al. [2] have closely investigated the flora and fauna of roadside verges. All of this referred literature shows that the effects of roads on wildlife are multiple, which can be positive or negative. The negative effects of roads on organisms are changing habitats, changing the environment's chemistry and physics, road kills, changing behaviour of animals, chemical pollution, acting as barrier and intensifying the dispersal of invasive and also exotic species $[15,7]$. However, roadside verges function as ecological corridors, serve as suitable habitat sources and conditions for organisms [17, 18, 19, 20, 21, 22, 7]. All of these potential effects are dependent on the complex interactions between species infrastructure and landscape [23, 24].

Owing to the ecological and biological features of beetles $[25,26]$, spiders [27], and woodlice [28, 29], they are excellent biological indicators. They can be also called hitchhiker insects as their spreading along roads has widely influenced the traffic. In addition to traffic, the quality of the habitat, the interactions between populations and the structure of the landscape also affect the spreading of arthropods and the composition of the communities [30, 31, 32, 33].

The main objective of this paper is to report on the spider, isopod, and ground beetle fauna of main road verges situated beside different habitat types that represent the main verge habitats of Hungary. Besides faunistic research, the effects of the different vegetation of main road verges on wildlife should also be investigated.

\section{Material and methods}

Along the Hungarian main roads, four sampling sites were selected, representing the main types of verge habitats. The first sampling area, Pilisjászfalú (Budapest-Esztergom, Pest County), was along Road No 10 and consisted of arid grassland with some small bushes shown in Fig. 1. The second sampling area, Mány (Budapest-Győr, Fejér County), was situated along Road No 1 between two roads in agricultural areas, in the lowlands and the hilly landscape of Hungary, shown in Fig. 2. The third sampling area, Herceghalom (Budapest-Györ, Pest County), was located along Road No1 between the road and the forest, shown in Fig. 3. The fourth sampling area, Agárd (Budapest-Székesfehérvár, Fejér County), was along Road No 7, crossing a wetland area in the western section of Lake Velence, shown in Fig. 4. 


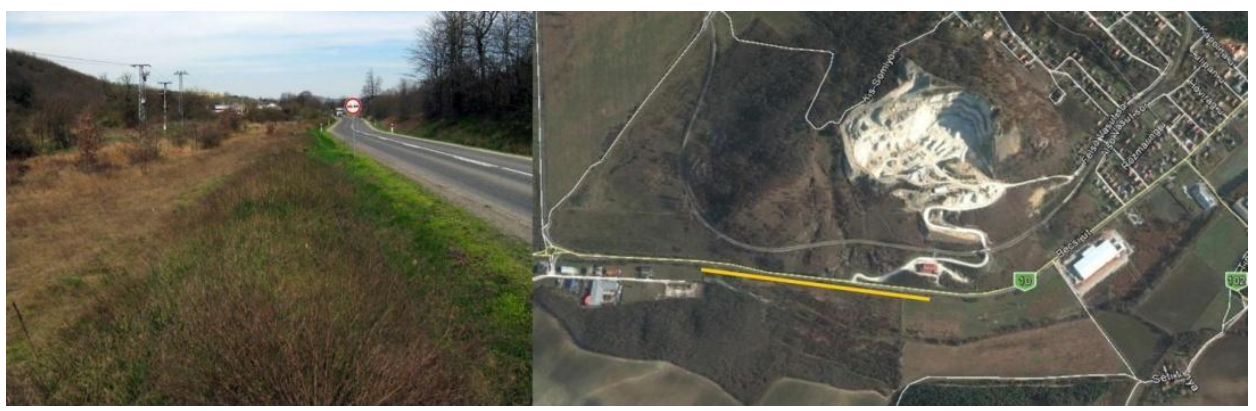

Figure 1. Pilisjászfalu sampling sites along Road No 10

Arthropods were sampled using 15-15 pitfall traps on each site and the distance between the traps was $5 \mathrm{~m}$. The 60 pitfall traps used in the study were transparent plastic cups filled with a $65 \%$ aqueous solution of ethylene glycol as a preservative fluid [34]. The traps were in the fields for three weeks, placed four times a year in different seasons (early spring, spring, summer, and autumn).

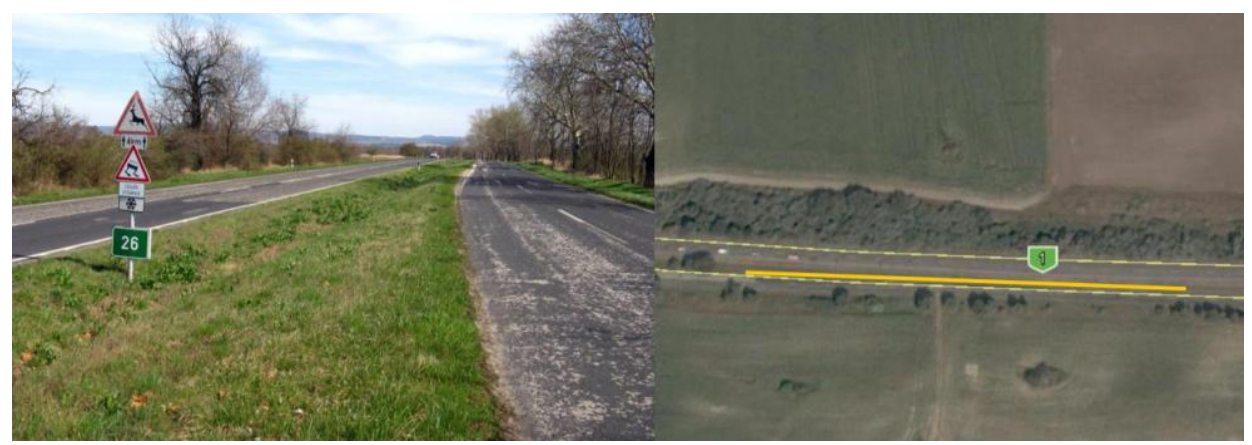

Figure 2. Mány sampling sites along Road No1

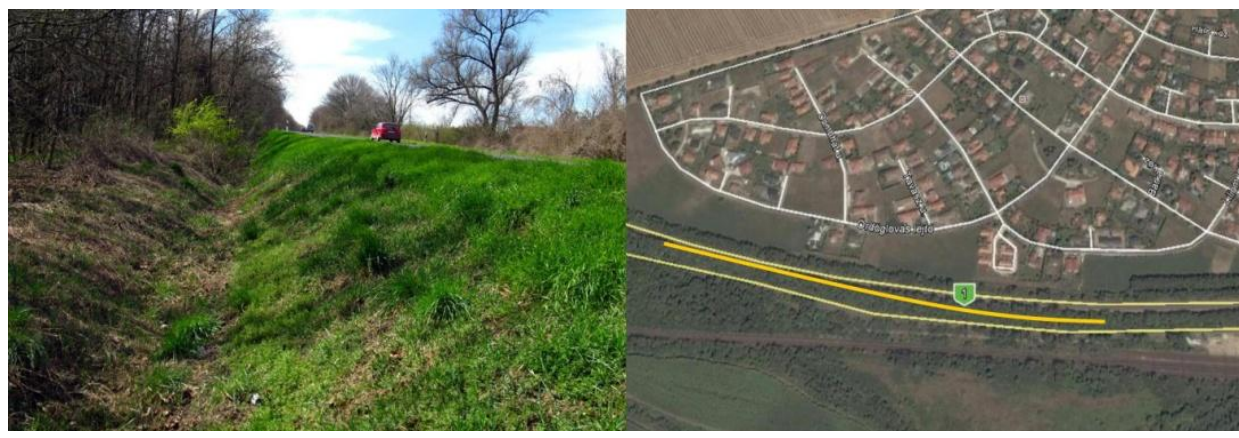

Figure 3. Herceghalom sampling sites along Road No1 


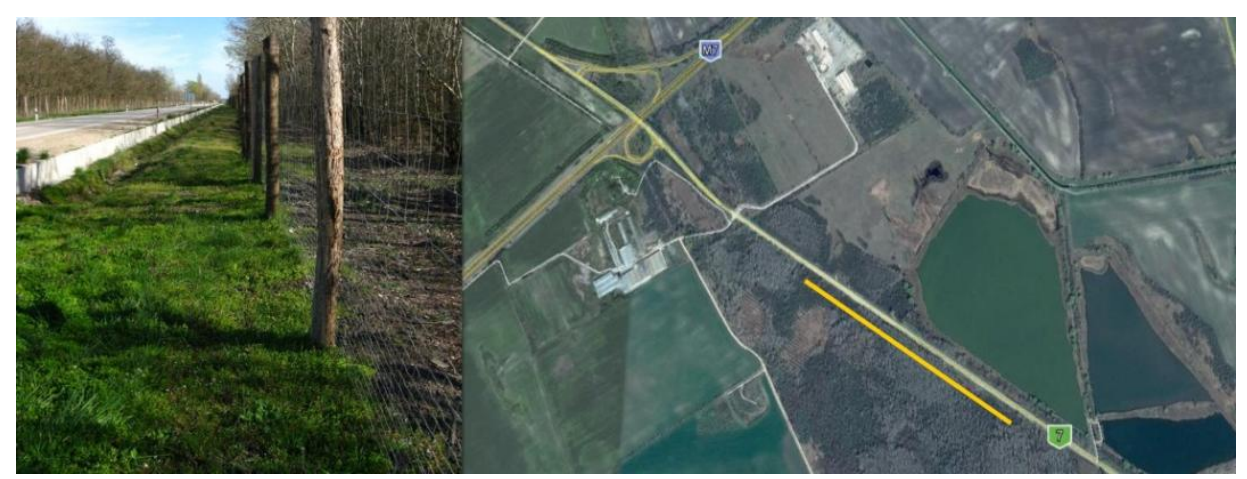

Figure 4. Agárd sampling sites along Road No 7

The characterizations of the ground-dwelling arthropod communities were based on species richness and abundance. For the analysis of the data, we used the PAST Paleontological Statistic suite. We counted Shannon-Wiener $(\mathrm{H})$ diversity for an accurate characterization of the examined isopod communities. We studied the species' preference for habitat using correspondence analysis.

\section{Results and discussion}

The sampling resulted 7,563 ground-dwelling arthropods from 168 species along main road verges. In total, 1,139 beetles from 83 species, 2,212 spiders from 81 species, and 4,212 isopods form 4 species were collected as shown in Table 1. Seven beetle species (Carabus convexus, Carabus coriaceus, Carabus germarii, Carabus granulatus, Carabus scheidleri, and Carabus violaceus) and one spider species (Nemesia pannonica) are included in the invertebrate Red List of Hungary [35]. The total Shannon-Wiener diversity was relatively high (2.69). On each verge, the Shannon-Wiener diversity of spiders was the highest (3.58), followed by beetles (3.32) and isopods (0.36).

In this study, beetles was the richest group of species. With regard to the size of beetles, the species can be grouped into small-sized species and medium-sized species [36]. Based on food reference, the omnivoreous or phytophagous and spermophagous species (Amara and Harpalus genus) were dominant [37]. According to wing development, the macropterous and dimorphous or polimorphous species (Amara and Harpalus genus) [36] were frequent. The majority of the species are common in Hungary, except the seven protected beetle species, among which $C$. cancellatus (42 individuals) was the dominant, followed by the $C$. coriaceus (36 individuals). Brachinus explodens (167 individuals) was recorded with the highest abundance, followed by Harpalus tardus (130 individuals) and Bembidion properans (100 individuals). H. tardus in arid 
grassland and the other two mentioned species in wetland were predominant. The sporadic Pterostichus elongatus benefits from wet sodic habitats [38, 39] and flies towards light [40]. P. incommodus is not a common species either [41], but its lifestyle is less known, except that it lives in open forests and wooded steppe [36]. In the Netherlands, Vermeulen [4] collected several rare carabid beetles on roadside verges. This and our results indicate that roadsides have a potential conservation value. In the case of beetles, wetland verges were prominent as they were found to dispose of the highest species richness and abundance as shown in fig-s 5, 6, and 7.

Lycosidae and Gnaphosidae of spider families were dominant. The highest species richness was observed in arid grassland verges and the abundance of spiders in the wetland verges was the highest as shown in fig-s 5, 6, and 7. Pardosa lugubris (263 individuals), Alopecosa trabalis (216 individuals), and Alopecosa pulverulenta (155 individuals) of spiders were observed as having the highest abundance.

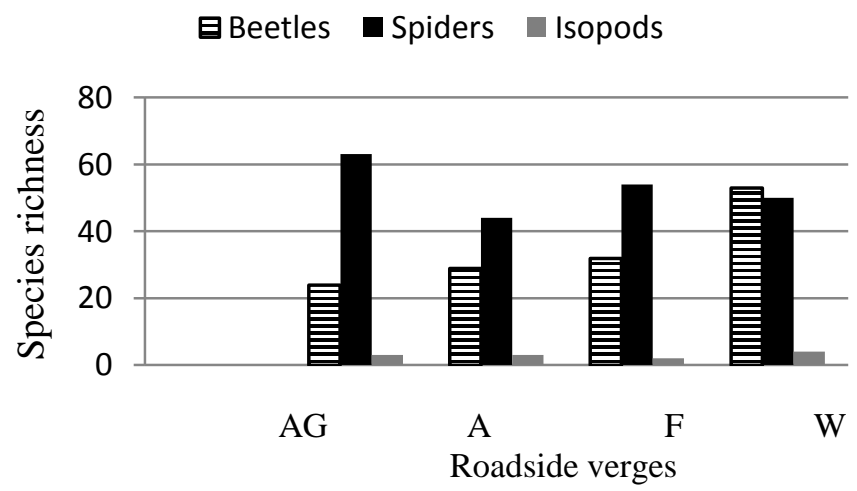

Figure 5. Species richness of ground-dwelling arthropods on the four roadside verges

In this regard, we have to mention Pardosa bifasciata (148 individuals), Xysticus kochi (147 individuals), and Pardosa agrestis (133 individuals). While $P$. lugubris, $P$. bifasciata, $P$. agrestis, and $X$. kochi dominated the wetland, A. trabalis was present in arid grassland and $A$. pulverulenta in agricultural landscapes with a high number of individuals. 


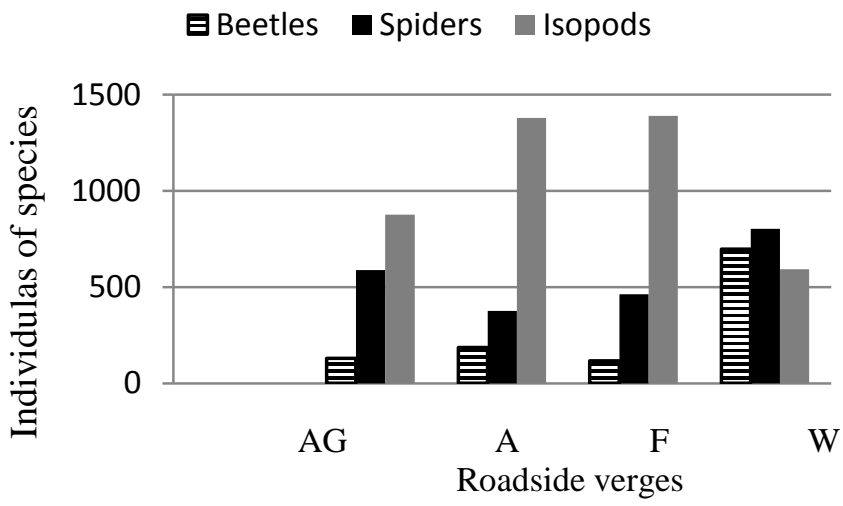

Figure 6. Individuals of species of ground-dwelling arthropods on the four roadside verges

Table 1. Distribution of species based on habitat types. Habitat types: AG: arid grassland, A: agricultural area, F: forest, W: wetland.

\begin{tabular}{|c|c|c|c|c|}
\hline \multirow{2}{*}{ Species } & \multicolumn{4}{|c|}{ Verges types } \\
\hline & $\mathbf{A G}$ & $\mathbf{A}$ & $\mathbf{F}$ & $\mathbf{W}$ \\
\hline Carabidae & & & & \\
\hline Abax parallelepipedus (Piller \& Mitterpacher, 1783) & & & $\mathrm{x}$ & \\
\hline Amara aenea (Degeer, 1774) & $\mathrm{X}$ & $\mathrm{x}$ & $\mathrm{x}$ & $\mathrm{X}$ \\
\hline Amara anthobia (A. et G. B. Villa, 1833) & $\mathrm{X}$ & & & $\mathrm{X}$ \\
\hline Amara apricaria (Paykull, 1790) & & $\mathrm{x}$ & & \\
\hline Amara consularis (Duftschmid, 1812) & & & $\mathrm{x}$ & \\
\hline Amara convexior (Stephens, 1828) & $\mathrm{X}$ & & $\mathrm{x}$ & \\
\hline Amara equestris (Duftschmid, 1812) & & $\mathrm{x}$ & & \\
\hline Amara familiaris (Duftschmid, 1812) & $\mathrm{X}$ & & $\mathrm{x}$ & $\mathrm{X}$ \\
\hline Amara lucida (Duftschmid, 1812) & & & & $\mathrm{X}$ \\
\hline Amara ovata (Fabricius, 1792) & & & $\mathrm{x}$ & \\
\hline Amara saphyrea (Dejean, 1828) & & & $\mathrm{x}$ & $\mathrm{X}$ \\
\hline Amara similata (Gyllenhal, 1810) & & & $\mathrm{x}$ & $\mathrm{X}$ \\
\hline Amara tibialis (Paykull, 1798) & & & & $\mathrm{X}$ \\
\hline Anchomenus dorsalis (Pontoppidan, 1763) & & $\mathrm{x}$ & $\mathrm{x}$ & $\mathrm{X}$ \\
\hline Anisodactylus binotatus (Fabricius, 1787) & & & & $\mathrm{X}$ \\
\hline Badister bullatus (Schrank, 1798) & & & $\mathrm{X}$ & \\
\hline Bembidion properans (Stephens, 1828) & & & & $\mathrm{X}$ \\
\hline Bembidion quadrimaculatum (Linnaeus, 1761) & & & & $\mathrm{X}$ \\
\hline Brachinus crepitans (Linnaeus, 1758) & & & $\mathrm{x}$ & $\mathrm{X}$ \\
\hline Brachinus explodens (Duftschmid, 1812) & & $\mathrm{x}$ & $\mathrm{x}$ & $\mathrm{X}$ \\
\hline Brachinus ganglbaueri (Apfelbeck, 1904) & & & & $\mathrm{X}$ \\
\hline
\end{tabular}




\begin{tabular}{|c|c|c|c|c|}
\hline \multirow{2}{*}{ Species } & \multicolumn{4}{|c|}{ Verges types } \\
\hline & $\mathbf{A G}$ & A & $\mathbf{F}$ & $\mathbf{W}$ \\
\hline Bradycellus harpalinus (Audinet-Serville, 1821) & & & & $\mathrm{x}$ \\
\hline Calathus ambiguous (Paykull, 1790) & & $\mathrm{x}$ & & $\mathrm{x}$ \\
\hline Calathus cinctus (Motschulsky, 1850) & & $\mathrm{x}$ & & \\
\hline Calathus fuscipes (Goeze, 1777) & & $\mathrm{x}$ & $\mathrm{x}$ & $\mathrm{x}$ \\
\hline Calathus melanocephalus (Linnaeus, 1758) & $\mathrm{x}$ & & & $\mathrm{x}$ \\
\hline Callistus lunatus (Fabricius, 1775) & $\mathrm{x}$ & & & \\
\hline Carabus cancellatus (Illiger, 1798) & & & & $\mathrm{x}$ \\
\hline Carabus convexus (Fabricius, 1775) & & & & $\mathrm{x}$ \\
\hline Carabus coriaceus (Linnaeus, 1758) & & $\mathrm{x}$ & $\mathrm{x}$ & $\mathrm{x}$ \\
\hline Carabus germarii (Sturm, 1815) & & & & $\mathrm{x}$ \\
\hline Carabus granulatus (Linnaeus, 1758) & & & & $\mathrm{x}$ \\
\hline Carabus scheidleri (Panzer, 1799) & $\mathrm{x}$ & & $\mathrm{x}$ & \\
\hline Carabus violaceus (Linnaeus, 1758) & & $\mathrm{x}$ & & \\
\hline Chlaenius festivus (Panzer, 1796) & & & & $\mathrm{x}$ \\
\hline Chlaenius nigricornis (Fabricius, 1787) & & & & $\mathrm{x}$ \\
\hline Chlaenius nitidulus (Schrank, 1781) & & & & $\mathrm{x}$ \\
\hline Chlaenius spp. & & & & $\mathrm{x}$ \\
\hline Dischyrius globosus (Herbst, 1784) & & & $\mathrm{x}$ & \\
\hline Harpalus affinis (Schrank, 1781) & & & & $\mathrm{x}$ \\
\hline Harpalus azureus (Fabricius, 1775) & & $\mathrm{x}$ & $\mathrm{x}$ & $\mathrm{x}$ \\
\hline Harpalus calceatus (Duftschmid, 1812) & & $\mathrm{x}$ & & \\
\hline Harpalus puncticeps (Stephens, 1828) & $\mathrm{x}$ & & & \\
\hline Harpalus cribricollis (Dejean, 1829) & & $\mathrm{x}$ & & \\
\hline Harpalus distinguendus (Duftschmid, 1812) & & & $\mathrm{x}$ & $\mathrm{x}$ \\
\hline Harpalus flavicornis (Dejean, 1829) & $\mathrm{x}$ & $\mathrm{x}$ & & $\mathrm{x}$ \\
\hline Harpalus griseus (Panzer, 1796) & $\mathrm{x}$ & & $\mathrm{x}$ & \\
\hline Harpalus pumilus (Sturm, 1818) & $\mathrm{x}$ & $\mathrm{x}$ & & \\
\hline Harpalus caspius roubali (Schauberger, 1928) & $\mathrm{x}$ & & & \\
\hline Harpalus rubripes (Duftschmid, 1812) & $\mathrm{x}$ & & & \\
\hline Harpalus sabulicola (Panzer, 1796) & & $\mathrm{x}$ & & \\
\hline Harpalus rufibarbis (Fabricius, 1792) & & & & $\mathrm{x}$ \\
\hline Harpalus rufipes (De Geer, 1774) & & $\mathrm{x}$ & $\mathrm{X}$ & $\mathrm{x}$ \\
\hline Harpalus serripes (Quensel in Schönherr, 1806) & & $\mathrm{x}$ & $\mathrm{x}$ & $\mathrm{x}$ \\
\hline Harpalus signaticornis (Duftschmid, 1812) & $\mathrm{x}$ & & & \\
\hline Harpalus smaragdinus (Duftschmid, 1812) & & $\mathrm{x}$ & & \\
\hline Harpalus tardus (Panzer, 1796) & $\mathrm{x}$ & $\mathrm{x}$ & $\mathrm{x}$ & $\mathrm{x}$ \\
\hline Leistus ferrugineus (Linnaeus, 1758) & $\mathrm{x}$ & $\mathrm{x}$ & $\mathrm{x}$ & $\mathrm{x}$ \\
\hline Microlestes maurus (Sturm, 1827) & & & & $\mathrm{x}$ \\
\hline Microlestes minutulus (Goeze, 1777) & & & & $\mathrm{x}$ \\
\hline Nebria brevicollis (Fabricius, 1792) & & & & $\mathrm{x}$ \\
\hline Notiophilus spp. & & & $\mathrm{x}$ & $\mathrm{x}$ \\
\hline
\end{tabular}




\begin{tabular}{|c|c|c|c|c|}
\hline \multirow{2}{*}{ Species } & \multicolumn{4}{|c|}{ Verges types } \\
\hline & AG & $\mathbf{A}$ & $\mathbf{F}$ & $\mathbf{W}$ \\
\hline Panagaeus bipustulatus (Fabricius, 1775) & $\mathrm{x}$ & & $\mathrm{x}$ & $\mathrm{x}$ \\
\hline Panagaeus crux-major (Linnaeus, 1758) & & & & $\mathrm{x}$ \\
\hline Parophonus complanatus (Dejean, 1829) & & $\mathrm{x}$ & & \\
\hline Parophonus maculicornis (Duftschmid, 1812) & & $\mathrm{x}$ & $\mathrm{x}$ & \\
\hline Platyderus rufus (Duftschmid, 1812) & & & $\mathrm{x}$ & \\
\hline Poecilus cupreus (Linnaeus, 1758) & $\mathrm{x}$ & & & $\mathrm{x}$ \\
\hline Poecilus Lepidus (Leske, 1785) & & & & $\mathrm{x}$ \\
\hline Poecilus sericeus (Fischer von Waldheim, 1823) & & $\mathrm{x}$ & & \\
\hline Poecilus versicolor (Sturm, 1824) & & & & $\mathrm{x}$ \\
\hline Pterostichus elongatus (Dufrschmid, 1812) & & & & $\mathrm{x}$ \\
\hline Pterostichus incommodus (Schaum, 1858) & & $\mathrm{x}$ & & \\
\hline Pterostichus melanarius (Illiger, 1798) & & & & $\mathrm{x}$ \\
\hline Pterostichus niger (Schaller, 1783) & & & & $\mathrm{x}$ \\
\hline Pterostichus vernalis (Panzer, 1795) & & & $\mathrm{x}$ & \\
\hline Stenolophus discophorus (Fischer von Waldheim, 1824) & & & & $\mathrm{x}$ \\
\hline Stenolophus mixtus (Herbst, 1784) & & & $\mathrm{x}$ & $\mathrm{x}$ \\
\hline Stenolophus teutonus (Schrank, 1781) & & & & $\mathrm{x}$ \\
\hline Syntomus pallipes (Dejean, 1825) & & $\mathrm{x}$ & & \\
\hline Tachys diabrachys bisbimaculatus (Chevrolat, 1860) & & & & $\mathrm{x}$ \\
\hline Trechus quadristriatus (Schrank, 1781) & $\mathrm{x}$ & $\mathrm{x}$ & & $\mathrm{x}$ \\
\hline Zabrus tenebrioides (Goeze, 1777) & $\mathrm{x}$ & $\mathrm{x}$ & & \\
\hline \multicolumn{5}{|l|}{ Araneae } \\
\hline \multicolumn{5}{|l|}{ Nemesiidae } \\
\hline Nemesia pannonica (Herman, 1879) & $\mathrm{x}$ & & & \\
\hline \multicolumn{5}{|l|}{ Segestriidae } \\
\hline \multicolumn{5}{|l|}{ Segestria senoculata (Linnaeus, 1758) } \\
\hline \multicolumn{5}{|l|}{ Dysderidae } \\
\hline Dysdera erythrina (Walckenaer, 1802) & $\mathrm{x}$ & $\mathrm{x}$ & $\mathrm{x}$ & \\
\hline Dysdera spp. juvenilis & $\mathrm{x}$ & $\mathrm{x}$ & $\mathrm{x}$ & \\
\hline Harpactea rubicunda (C. L. Koch, 1838) & $\mathrm{x}$ & $\mathrm{x}$ & $\mathrm{x}$ & \\
\hline \multicolumn{5}{|l|}{ Mimetidae } \\
\hline Ero furcata (Villers, 1789) & & & $\mathrm{x}$ & \\
\hline \multicolumn{5}{|l|}{ Theridiidae } \\
\hline Asagena phalerata (Panzer, 1801) & $\mathrm{x}$ & $\mathrm{x}$ & $\mathrm{x}$ & $\mathrm{x}$ \\
\hline Enoplognatha thoracica (Hahn, 1833) & & $\mathrm{x}$ & $\mathrm{x}$ & \\
\hline Euryopis flavomaculata (C. L. Koch, 1836) & $\mathrm{x}$ & & & \\
\hline Euryopis quinqueguttata (Thorell, 1875) & & & $\mathrm{x}$ & \\
\hline Steatoda albomaculata (De Geer, 1778) & & $\mathrm{x}$ & & $\mathrm{x}$ \\
\hline \multicolumn{5}{|l|}{ Linyphiidae } \\
\hline Centromerus sylvaticus (Blackwall, 1841) & $\mathrm{x}$ & & $\mathrm{x}$ & $\mathrm{x}$ \\
\hline Diplostyla concolor (Wider, 1834) & & & $\mathrm{x}$ & $\mathrm{x}$ \\
\hline
\end{tabular}




\begin{tabular}{|c|c|c|c|c|}
\hline \multirow{2}{*}{ Species } & \multicolumn{4}{|c|}{ Verges types } \\
\hline & $\mathbf{A G}$ & $\mathbf{A}$ & $\mathbf{F}$ & $\mathbf{W}$ \\
\hline Erigone dentipalpis (Wider, 1834) & & & $\mathrm{X}$ & $\mathrm{x}$ \\
\hline Megalepthyphantes nebulosus (Sundevall, 1830) & & & $\mathrm{X}$ & \\
\hline Prinerigone vagans (Audouin, 1826) & & & & $\mathrm{X}$ \\
\hline Stemonyphantes lineatus (Linnaeus, 1758) & $\mathrm{X}$ & & & \\
\hline \multicolumn{5}{|l|}{ Tetragnathidae } \\
\hline Pachygnatha degeeri (Sundevall, 1830) & & & $\mathrm{x}$ & $\mathrm{X}$ \\
\hline \multicolumn{5}{|l|}{ Araneidae } \\
\hline Agelenatea redii (Scopoli, 1763) & $\mathrm{x}$ & & & \\
\hline Araneus diadematus (Clerck, 1757) & & & $\mathrm{x}$ & \\
\hline Argiope bruennichi (Scopoli, 1772) & $\mathrm{x}$ & & & \\
\hline Cercidia prominens (Westring, 1851) & & & $\mathrm{x}$ & \\
\hline \multicolumn{5}{|l|}{ Lycosidae } \\
\hline Alopecosa accentuata (Latreille, 1817) & $\mathrm{x}$ & & & \\
\hline Alopecosa cuneata (Clerck, 1757) & $\mathrm{x}$ & $\mathrm{x}$ & & $\mathrm{x}$ \\
\hline Alopecosa pulverulenta (Clerck, 1757) & $\mathrm{X}$ & $\mathrm{x}$ & $\mathrm{X}$ & $\mathrm{x}$ \\
\hline Alopecosa sulzeri (Pavesi, 1873) & $\mathrm{x}$ & & & \\
\hline Alopecosa trabalis (Clerck, 1757) & $\mathrm{x}$ & $\mathrm{x}$ & $\mathrm{X}$ & $\mathrm{x}$ \\
\hline Alopecosa spp. juv & $\mathrm{x}$ & $\mathrm{x}$ & $\mathrm{X}$ & $\mathrm{x}$ \\
\hline Arctosa leopardus (Sundevall, 1833) & & & & $\mathrm{x}$ \\
\hline Aulonia albimana (Walckenaer, 1805) & $\mathrm{x}$ & $\mathrm{x}$ & $\mathrm{x}$ & $\mathrm{x}$ \\
\hline Hogna radiata (Latreille, 1819) & $\mathrm{x}$ & $\mathrm{x}$ & & $\mathrm{x}$ \\
\hline Pardosa agrestis (Westring, 1861) & $\mathrm{x}$ & $\mathrm{x}$ & $\mathrm{X}$ & $\mathrm{X}$ \\
\hline Pardosa amentata (Clerck, 1757) & & & $\mathrm{X}$ & \\
\hline Pardosa bifasciata (C. L. Koch, 1834) & $\mathrm{x}$ & $\mathrm{x}$ & $\mathrm{X}$ & $\mathrm{x}$ \\
\hline Pardosa lugubris (Walckenaer, 1802) & $\mathrm{x}$ & $\mathrm{x}$ & $\mathrm{X}$ & $\mathrm{X}$ \\
\hline Pardosa paludicola (Clerck, 1757) & & & $\mathrm{X}$ & $\mathrm{x}$ \\
\hline Pardosa proxima (C. L. Koch, 1847) & & & & $\mathrm{x}$ \\
\hline Pardosa riparia (C. L. Koch, 1833) & $\mathrm{X}$ & $\mathrm{x}$ & $\mathrm{X}$ & $\mathrm{x}$ \\
\hline Pardosa spp. juv & $\mathrm{x}$ & $\mathrm{x}$ & $\mathrm{X}$ & \\
\hline Trochosa robusta (Simon, 1876) & $\mathrm{x}$ & $\mathrm{x}$ & $\mathrm{X}$ & \\
\hline Trochosa terricola (Thorell, 1856) & $\mathrm{X}$ & $\mathrm{x}$ & $\mathrm{X}$ & $\mathrm{x}$ \\
\hline Trochosa spp. juv & $\mathrm{x}$ & $\mathrm{x}$ & $\mathrm{x}$ & $\mathrm{x}$ \\
\hline Xerolycosa miniata (C. L. Koch, 1834) & & & & $\mathrm{X}$ \\
\hline Xerolycosa nemoralis (Westring, 1861) & & & & $\mathrm{x}$ \\
\hline \multicolumn{5}{|l|}{ Pisauridae } \\
\hline Pisaura mirabilis (Clerck, 1757) & $\mathrm{x}$ & $\mathrm{x}$ & $\mathrm{x}$ & $\mathrm{x}$ \\
\hline \multicolumn{5}{|l|}{ Agelenidae } \\
\hline Allagelena gracilens $(\mathrm{C} . \mathrm{L} . \mathrm{Koch}, 1841)$ & $\mathrm{X}$ & & $\mathrm{x}$ & $\mathrm{X}$ \\
\hline Coelotes terrestris (Wider, 1834) & & & $\mathrm{X}$ & \\
\hline Eratigena agrestis (Walckenaer, 1802) & & $\mathrm{X}$ & $\mathrm{X}$ & $\mathrm{X}$ \\
\hline Urocoras longispinus (Kulczynski, 1897) & $\mathrm{x}$ & $\mathrm{x}$ & $\mathrm{X}$ & \\
\hline
\end{tabular}




\begin{tabular}{|c|c|c|c|c|}
\hline \multirow{2}{*}{ Species } & \multicolumn{4}{|c|}{ Verges types } \\
\hline & AG & $\mathbf{A}$ & $\mathbf{F}$ & $\mathbf{W}$ \\
\hline \multicolumn{5}{|l|}{ Titanoecidae } \\
\hline Titanoeca quadriguttata (Hahn, 1833) & & $\mathrm{x}$ & & \\
\hline Titanoeca shineri (L. Koch, 1872) & & $\mathrm{x}$ & & \\
\hline \multicolumn{5}{|l|}{ Eutichuridae } \\
\hline Cheiracanthium virescens (Sundevall, 1833) & $\mathrm{x}$ & & & \\
\hline \multicolumn{5}{|l|}{ Miturgidae } \\
\hline Zora spinimana (Sundevall, 1833) & $\mathrm{x}$ & $\mathrm{x}$ & $\mathrm{x}$ & \\
\hline \multicolumn{5}{|l|}{ Anyphaenidae } \\
\hline Anyphaena accentuata (Walckenaer, 1802) & & $\mathrm{x}$ & $\mathrm{x}$ & \\
\hline \multicolumn{5}{|l|}{ Liocranidae } \\
\hline Agroeca cuprea (Menge, 1873) & $\mathrm{x}$ & & & $\mathrm{x}$ \\
\hline Liocranoeca striata (Kulczynski, 1882) & & & & $\mathrm{x}$ \\
\hline Scotina celans (Blackwall, 1841) & & & $\mathrm{x}$ & \\
\hline \multicolumn{5}{|l|}{ Phrurolithidae } \\
\hline Phrurolithus festivus (C. L. Koch, 1835) & $\mathrm{x}$ & $\mathrm{X}$ & & \\
\hline \multicolumn{5}{|l|}{ Clubionidae } \\
\hline Clubiona pseudoneglecta (Wunderlich, 1994) & $\mathrm{x}$ & & & \\
\hline Clubiona terrestris (Westring, 1851) & & & $\mathrm{x}$ & \\
\hline \multicolumn{5}{|l|}{ Zodariidae } \\
\hline Zodarion germanicum (C. L. Koch, 1837) & $\mathrm{x}$ & & & \\
\hline Zodarion rubidium (Simon, 1914) & $\mathrm{x}$ & & & $\mathrm{x}$ \\
\hline Zodarion spp. juv & $\mathrm{x}$ & & & \\
\hline \multicolumn{5}{|l|}{ Gnaphosidae } \\
\hline Drassodes cupreus (Blackwall, 1834) & & $\mathrm{X}$ & & \\
\hline Drassodes lapidosus (Walckenaer, 1802) & & $\mathrm{x}$ & & \\
\hline Drassodes pubescens (Thorell, 1856) & $\mathrm{x}$ & $\mathrm{x}$ & $\mathrm{x}$ & $\mathrm{x}$ \\
\hline Drassyllus praeficus (L. Koch, 1866) & $\mathrm{x}$ & $\mathrm{x}$ & $\mathrm{x}$ & $\mathrm{x}$ \\
\hline Drassyllus pusillus (C. L. Koch, 1833) & & $\mathrm{x}$ & & $\mathrm{x}$ \\
\hline Drassyllus villicus (Thorell, 1875) & & $\mathrm{x}$ & $\mathrm{x}$ & \\
\hline Drassyllus spp. juv & $\mathrm{x}$ & $\mathrm{x}$ & $\mathrm{x}$ & $\mathrm{x}$ \\
\hline Gnaphosa lucifuga (Walckenaer, 1802) & & & $\mathrm{x}$ & \\
\hline Haplodrassus signifer (C. L. Koch, 1839) & $\mathrm{x}$ & $\mathrm{x}$ & $\mathrm{x}$ & $\mathrm{x}$ \\
\hline Haplodrassus umbratilis (L. Koch, 1866) & & & & $\mathrm{x}$ \\
\hline Trachyzelotes pedestris (C. L. Koch, 1837) & $\mathrm{x}$ & $\mathrm{X}$ & $\mathrm{x}$ & $\mathrm{x}$ \\
\hline Zelotes apricorum (L. Koch, 1876) & $\mathrm{x}$ & & $\mathrm{x}$ & $\mathrm{x}$ \\
\hline Zelotes electus (C. L. Koch, 1839) & $\mathrm{x}$ & $\mathrm{X}$ & $\mathrm{x}$ & $\mathrm{x}$ \\
\hline Zelotes latreillei (Simon, 1878) & $\mathrm{x}$ & & $\mathrm{x}$ & $\mathrm{x}$ \\
\hline Zelotes longipes (L. Koch, 1866) & $\mathrm{x}$ & & $\mathrm{x}$ & \\
\hline Zelotes petrensis (C. L. Koch, 1839) & $\mathrm{x}$ & & & $\mathrm{x}$ \\
\hline Zelote spp. juv & $\mathrm{x}$ & & $\mathrm{x}$ & $\mathrm{x}$ \\
\hline Philodromidae & & & & \\
\hline
\end{tabular}




\begin{tabular}{|c|c|c|c|c|}
\hline \multirow{2}{*}{ Species } & \multicolumn{4}{|c|}{ Verges types } \\
\hline & $\mathbf{A G}$ & $\mathbf{A}$ & $\mathbf{F}$ & $\mathbf{W}$ \\
\hline Thanatus arenarius (Thorell, 1872) & $\mathrm{x}$ & $\mathrm{x}$ & & \\
\hline Thanatus formicinus (Clerck, 1757) & $\mathrm{X}$ & & & \\
\hline Thanatus spp. juvenilis & $\mathrm{x}$ & $\mathrm{x}$ & $\mathrm{x}$ & $\mathrm{X}$ \\
\hline Tibellus spp. juvenilis & $\mathrm{x}$ & & & $\mathrm{X}$ \\
\hline \multicolumn{5}{|l|}{ Thomisidae } \\
\hline Ozyptila atomaria (Panzer, 1801) & & $\mathrm{x}$ & $\mathrm{x}$ & \\
\hline Ozyptila claveata (Walckenaer, 1837) & $\mathrm{X}$ & $\mathrm{x}$ & $\mathrm{X}$ & \\
\hline Ozyptila praticola (C. L. Koch, 1837) & & $\mathrm{x}$ & & $\mathrm{X}$ \\
\hline Ozyptila scabricula (Westring, 1851) & $\mathrm{x}$ & & & \\
\hline Ozyptila simplex (O. P.-Cambridge, 1862) & $\mathrm{X}$ & & & $\mathrm{X}$ \\
\hline Xysticus acerbus (Thorell, 1872) & $\mathrm{x}$ & & & \\
\hline Xysticus cristatus (Clerck, 1857) & $\mathrm{X}$ & & & $\mathrm{X}$ \\
\hline Xysticus luctator (L. Koch, 1870) & & & & $\mathrm{X}$ \\
\hline Xysticus kochi (Thorell, 1872) & $\mathrm{x}$ & $\mathrm{x}$ & $\mathrm{X}$ & $\mathrm{X}$ \\
\hline Xysticus spp. juvenilis & $\mathrm{X}$ & $\mathrm{X}$ & $\mathrm{X}$ & $\mathrm{X}$ \\
\hline \multicolumn{5}{|l|}{ Salticidae } \\
\hline Euophrys spp. juv & $\mathrm{x}$ & & & \\
\hline Heliophanus cupreus (Walckenaer, 1802) & & & $\mathrm{x}$ & \\
\hline Pellenes tripunctatus (Walckenaer, 1802) & $\mathrm{x}$ & & & \\
\hline Phlegra fasciata (Hahn, 1826) & $\mathrm{x}$ & & & \\
\hline Salticus ssp. juvenilis & $\mathrm{X}$ & & & \\
\hline \multicolumn{5}{|l|}{ Isopods } \\
\hline \multicolumn{5}{|l|}{ Trachelipodidae } \\
\hline Porcellium collicola (Verhoeff, 1907) & $\mathrm{X}$ & $\mathrm{x}$ & $\mathrm{x}$ & $\mathrm{X}$ \\
\hline Trachelipus rathkii (Brandt, 1833) & $\mathrm{x}$ & & & $\mathrm{X}$ \\
\hline \multicolumn{5}{|l|}{ Porcellionideae } \\
\hline Porcelliodides pruinosus (Brandt, 1833) & & $\mathrm{x}$ & & $\mathrm{X}$ \\
\hline \multicolumn{5}{|l|}{ Armadillidiidae } \\
\hline Armadillidium vulgare (Latreille, 1804) & $\mathrm{x}$ & $\mathrm{x}$ & $\mathrm{X}$ & $\mathrm{X}$ \\
\hline
\end{tabular}

Only the arid grassland provides suitable habitats for the one registered protected species: Nemesia pannonica, which is one of the most valuable (10 000 HUF) nature conservation spider species in Hungary. The single Hungarian representative of the Nemesiidae family is submediterranean-distributed. Last year, a growing number of occurrences of this species were registered, such as in Pilis [42], East Mezöföld [43], and Mátra [44]. The presence of this species with special needs confirms that main road verges occasionally expand the area of nature reserves [4]. Web spiders (Agelenidae and Araneidae family) mainly selected the verges' borders with forests and meadows, which provide suitable conditions for spiders to make web. Crab spiders, generally waiting to prey on colourful flowers, were found near grasslands. 
These findings demonstrate the importance of mowing for floral diversity [45, 46]. Each of the four registered ant-specialist species (Zodarion rubidum, Zodarion germanicum, Asagena phalerata, and Thanatus arenarius) was identified on arid grasslands as well and the A. phalerata stands out from among the others with its high abundance and incidence rate. Just few individuals of the rare Drassodes cupreus, Coelotes terrestris, and the previously mentioned ant specialist $Z$. rubidum were observed, and they did not show any habitat preference. The ant-specialist species show that ant as pioneer species are highly abundant on main road verges.

In this study, isopods proved to be the most poorly represented group of species. The highest abundance of species was found on the forested verges, but we also found the lowest species richness there as shown in fig-s 5 and 6.The ordination statistics demonstrates well that woodlice species prefer the wetland to other regions as shown in Fig. 7.
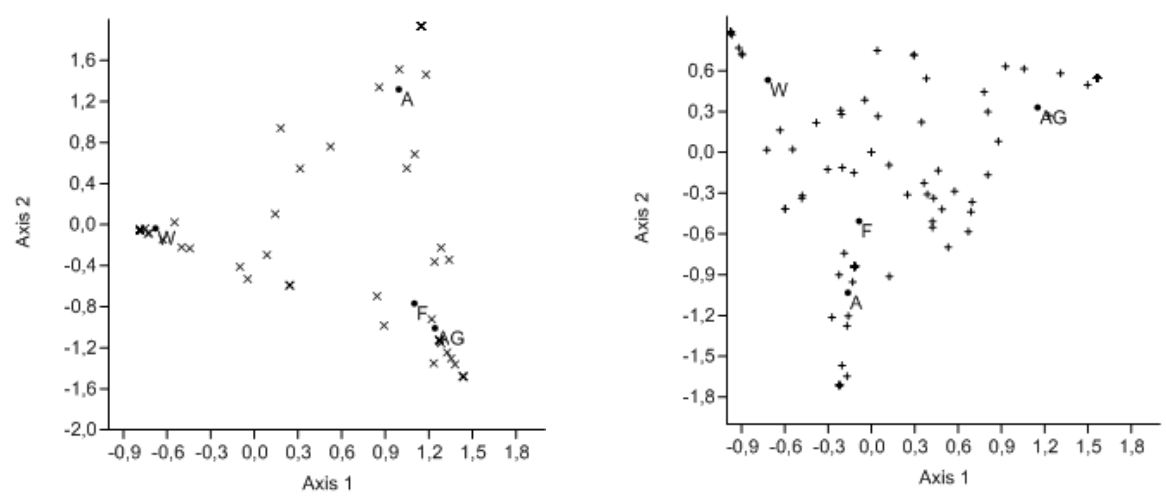

A

B

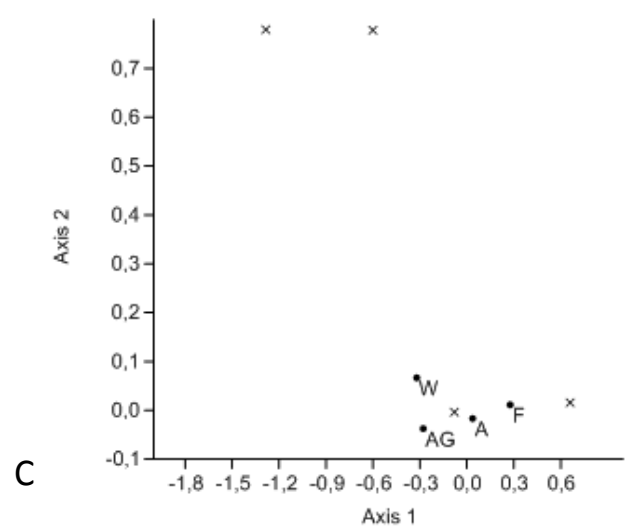

Figure 7. Correspondence analysis of carabidae (A), aranea (B), and isopod (C) species 
Each of the four registered isopod species are habitat generalists - they are able to successfully adapt to most continental habitats, whether natural or not. In all of the four investigated sites, Armadillidium vulgare of the isopod species was the most prevalent, followed by Porcellium collicola. Both of these species were present with a high abundance in forested and agricultural verges. The typical indicator of anthropogenic impacts, the cosmopolitan A. vulgare, can be found in most habitats except for protected hardwood forests [47]. P. collicola is one of the most frequent species in Hungary - found especially in parks and gardens, it can also be detected in most continental landscapes, excepting densely populated urban areas and protected hardwood forests [47]. Few individuals of Trachelipus rathkii and Porcelliodides pruinosus occurred in only two types of main road verges. T. rathkii is a common species in Hungary [48]: it can be observed near synanthropic environments, moderately humid habitats, and river valleys $[49,50]$. P. pruinosus is a cosmopolitan, widely spread species in Europe [51] and it can be found in synanthropic habitats indicating active human influence [52]. We expected the presence of exotic species because during our previous studies carried out along highways $[13,14]$ we have experienced that highway verges contribute to the creation of new habitats for several alien species. The explanation for this might be that highway networks are very different from lower-level roads [53, 54] and the number of sampling sites on main roads was different from the case of the highways.

\section{Conclusions}

Our study has clearly demonstrated that main road verges prove to be suitable habitats and provide necessary conditions for several carabid beetle and spider species, but isopods do not benefit from this landscape type. Roadside verges are frequently connected with natural reserves and urban habitats, allowing the spreading of species between them. These verges function as invasion pathways for arthropod species, but are also refuges for protected and endangered species in agriculturedominated landscapes in Hungary. The different surrounding vegetation of the examined main road stretches substantially affects the diversity and the spreading of ground-dwelling arthropods. The observed high diversity of arthropods reflects that the main road verges are important in terms of species richness.

\section{Acknowledgements}

We thank the National Road Authority and the field experts for botanical and zoological reference sampling and for measuring the biotic and abiotic environmental parameters of each sampling area. Our project was funded by CEDR-Harmony. 


\section{References}

[1] Prunier, J. G., Kaufmann, B., Fenet, S., Picard, D., Pompanon, F., Joly, P., Lena, J. P. (2013), Optimizing the trade-off between spatial and genetic sampling efforts in patchy populations: towards a better assessment of functional connectivity using an individual-based sampling scheme. Mol. Ecol. Res. 22(22), 5516-5530.

[2] Forman, R. T. T., Sperling, D., Bisonete, J. A., Clevenger, A. P. (eds). (2003), Road ecology: science and solutions. Island Press Washington, Covelo, London.

[3] Noordijk, J., Schaffers, A. P., Sykora, K. V. (2008), Diversity of ground beetles (Coleoptera: Carabidae) and spiders (Araneae) in roadside verges with grey hair-grass vegetation. Eur. $J$. Entomol. 105, 257-265.

[4] Vermeulen, H. J. W. (1993), The composition of the carabid fauna on poor sandy road-side verges in relation to comparable open areas. Biodivers. Conserv. 2, 331-350.

[5] Vermeulen, W., Vuuren, A. J., Chipoulet, M., Schaeffer, L., Appeldoorn, E., Weeda, G., Jaspers, N. G., Priestley, A., Arlett, C. F., Lehmann, A. R. (1994), Three unusual repair deficiencies associated with transcription factor BTF2 (TFIIH): evidence for the existence of a transcription syndrome. Cold Spring Harb. Symp. Quant. Biol. 59, 317-329.

[6] Mader H. J. (1984), Animal habitat isolation by roads and agricultural fields. Conserv. Biol. 29, 81-96.

[7] Knapp, M., Saska, P., Knappová, J., Vonička, P., Moravec, P., Kurka, A., Andel, P. (2013), The habitat-specific effects of highway proximity on ground-dwelling arthropods: implications for biodiversity conservation. Biol. Conserv. 164, 22-29.

[8] Koivula, M. J., Vermuelen, H. J. W. (2005), Highways and forest fragmentation effects on carabid beetles (Coleoptera, Carabidae). Landsc. Ecol. 20, 911-926.

[9] Koivula, M., Kotze, D. J., Salokannel, J. (2005), Beetles (Coleoptera) in central reservations of three city highway roads around the city of Helsinki, Finland. Ann. Zool. Fenn. 42, 615-626.

[10] Le Viol, I., Julliard, R., Kerbiriou, C., Redon, L., Carnino, N., Machon, N., Porcher, E. (2008), Plant and spider communities benefit differently from the presence of planted hedgerows in highway verges. Biol. Conserv.141(6), 1581-1590.

[11] Muskett, C. J., Jones, M. P. (1980), The dispersal of lead, cadmium and nickel from motor vehicles and effects on roadside invertebrate macrofauna. Environ. Pollut. Ecological and Biological 23(3), 231-242.

[12] Vona-Túri, D., Szmatona-Túri, T. (2012), Adatok a Mátra-hegység ászkarák (Crustacea: Isopoda: Oniscidea) faunájához, különös tekintettel az útmenti élőhelyekre. Termvéd. Közl. 18, 537-548.

[13] Vona-Túri, D., Szmatona-Túri, T., Kiss, B. (2013), Szárazföldi ászkarák együttesek (Crustacea: Isopoda: Oniscidea) a magyarországi autópályák szegélyzónájában. Termvéd. Közl. 19, 106116.

[14] Vona-Túri, D., Szmatona-Túri, T., Kiss, B. (2015), Autópályák szárazföldi ászkarákegyütteseinek (Crustacea: Isopoda: Oniscidea) ökológiai és diverzitás vizsgálata. Termvéd. Közl. 21, 395-406.

[15] Trombulak, S. C., Frissell, C. A. (2000), Review of ecological effects of roads on terrestrial and aquatic communities. Conserv Biol. 14(1), 18-30.

[16] Holderegger, R., Di Giulio, M. (2010), The genetic effects of roads: a review of empirical evidence. Basic. Appl. Ecol. 11(6), 522-531.

[17] Tikka, P. M., Hogmander, H., Koski, P. S. (2001), Road and railway verges serve as dispersal corridors for grassland plants. Landsc. Ecol. 16(7), 659-666.

[18] Kiss, B., Lengyel, G., Nagy, Zs., Kárpáti, Zs. (2013), A pettyesszárnyú muslica (Drosophila suzukii) első Magyarországi előfordulása. Növényvéd. 49(3), 97-99. 
[19] Koczor, S., Kiss, B., Szita, É., Fetykó, K. (2012), Two leafhopper species new to the fauna of Hungary (Hemiptera: Cicadomorpha: Cicadellidae). Acta Phytopathol. Entomol. Hung. 47(1), 69-73.

[20] Kontschán, J., Kiss, B. (2013), Egy ritka takácsatka, a Petrobia latens (Müller, 1776) második igazolt előfordulása Magyarországon (Acari: Tetranychidae). Növényvéd. 49, 281-284.

[21] Kozár, F., Szentkirályi, F., Kádár, F., Bernáth, B. (2004), Éghajlatváltozás és a rovarok. "AGRO-21" Füzetek 33, 49-64.

[22] Kozár, F., Szita, É., Fetykó, K., Neider, D., Konczné Benedicty, Zs., Kiss, B. (2013), Pajzstetvek, sztrádák, klíma. MTA ATK Növényvédelmi Intézet, Budapest.

[23] Woltz, H. W., Gibbs, J. P., Ducey, P. K. (2008), Roadcrossing structures for amphibians and reptiles: informing design through behavioral analysis. Biol. Conserv. 141, 2745-2750.

[24] Balkenhol, N., Waits, L. P. (2009), Molecular road ecology: exploring the potential of genetics for investigating transportation impacts on wildlife. Mol. Ecol. Res. 18(20), 4151-4164.

[25] Lövei, G. L., Sunderland, K. D. (1996), Ecology and behavior of ground beetles (Coleoptera: Carabidae). Annu. Rev. Entomol. 41, 231-256.

[26] Pearce, J. L., Venier, L. A. (2006), The use of ground beetles (Coleoptera: Carabidae) and spiders (Araneae) as bioindicators of sustainable forest management: A review. Ecol. Indic. 6, 780-793.

[27] Horváth, R., Magura, T., Szinetár, Cs., Tóthmérész, B. (2009), Spiders are not less diverse in small and isolated grasslands, but less diverse in overgrazed grasslands; a field study (East Hungary, Nyirseg). Agric. Ecosyst. Environ.130, 16-22.

[28] Dallinger, R., Berger, B., Birkel, S. (1992), Terrestrial isopods: useful bioindicators of urban metal pollution. Oecologia 89, 32-41.

[29] Paoletti, M. G., Hassall, M. (1999), Woodlice (Isopoda, Oniscidea): their potential for assessing sustainability and use as bioindicators. Agr. Ecosyst. Environ. 74, 157-165.

[30] Schmitz, O. J., Hamback, P. A., Beckerman, A. P. (2000), Trophic cascades in terrestrial systems: a review of the effects of carnivore removals on plants. Am. Nat. 155, 141-153.

[31] With, K. A., Pavuk, D. M., Worchuck, J. L., Oates, R. K., Fisher, J. L. (2002), Threshold effects of landscape structure on biological control in agroecosystems. Ecol. Appl. 12, 52-65.

[32] Jeanneret, Ph., Schüpbach, B., Pfiffner, L., Herzog, F., Walter, Th. (2003), The Swiss agrienvironmental programme and its effects on selected biodiversity indicators. J. Nat. Conserv.11, 213-220.

[33] Jeanneret, Ph., Schüpbach, B., Pfiffner, L., Walter, T. (2003), Arthropod reaction to landscape and habitat features in agricultural landscapes. Landsc. Ecol. 18, 253-263.

[34] Kádár, F., Samu, F. (2006), A duplaedényes talajcsapdák használata Magyarországon (On the initial implementation and use of double-cup pitfall traps in Hungary). Növényvéd. 42, 305312.

[35] Rakonczay, Z. (ed.). (1989), Vörös Könyv. Akadémiai Kiadó, Budapest.

[36] Hurka, K. (1996), Carabidae of the Czech and Slovak Republics. Kabourek, Zlin, Czech Republic.

[37] Bertrandi, F., Zetto Brandmayr, T. (1991), Osservazioni sulla dieta e cenni sulla bionomia del genere Harpalus Latreille (Coleoptera, Carabidae), Ber. nat.-med. Verein Innsbruck 78, 145155.

[38] Szél, Gy., Bérces, S. (2002), Carabidae (Coloptera) from the Fertő-Hanság National Park, in The Fauna of the Fertö-Hanság National Park, II. Magyar Természettudományi Múzeum, Budapest, 379-399.

[39] Kutasi, Cs. (2004), A Kab-hegy környéki tavak és láprétek futóbogarai (Col.: Carabidae), Fol. Mus. hist.-nat. bakonyiensis 21, 97-110. 
[40] Kádár, F., Szél, Gy., Retezár, I., Kutasi, Cs. (2005), New records of ground beetles (Coleoptera: Carabidae) attracted to light traps in Hungary. In: European Carabidology 2003. Proc. $11^{\text {th }}$ European Carabidologists' Meeting. DIAS Report Plant Production 114, 137-144.

[41] Kutasi, Cs., Szél, Gy. (2008), A Pterostichus melas (CREUTZER, 1799), a P. hungaricus (DEJEAN, 1828) és a P. incommodus (SCHAUM, 1858) előfordulása a Bakonyban. Fol. Mus. hist.-nat. bakonyiensis 25, 55-63.

[42] Samu, F. (2007), Pókok szünbiológiai kutatása az ember által befolyásolt tájban. Akadémiai Doktori Értekezés, MTA Növényvédelmi Kutatóintézet, Budapest.

[43] Szinetár Cs. Kutatási jelentés. http://pannongyep.hu/dok-letolt/ LIFEBelsobarand_Pokok_ SzCs2008hu.pdf.

[44] Szmatona-Túri, T., Vona-Túri, D. (2012), A magyar aknászpók (Nemesia pannonica Herman, 1879) újabb előfordulása Magyarországon. Termvéd. Közl. 18, 480-486.

[45] Buttler, A. (1992), Permanent plot research in wet meadows and cutting experiment. Vegetatio $103,113-124$.

[46] Güsewell, S., Buttler, A., Klötzli, F. (1998), Short-term and long-term effects of mowing on the vegetation of two calcareous fens. J. Veg. Sci. 9, 861-872.

[47] Farkas, S., Vilisics, F. (2013), Magyarország szárazföldi ászkarák faunájának határozója (Isopoda: Oniscidea). Nat. Somogy. 23, 89-124.

[48] Farkas, S. (1998), Population dynamics, spatial distribution, and sex ratio of Trachelipus rathkei Brandt (Isopoda: Oniscidea) in a wetland forest by the Drava river. Isr. J. Zool. 44, 323-331.

[49] Snider, R., Shaddy, H. (1980), The ecobiology of Trachelipus rathkii (Isopoda). Pedobiol. (Jena) 20, 394-410.

[50] Forró, L., Farkas, S. (1998), Checklist, preliminary distribution maps, and bibliography of woodlice in Hungary (Isopoda: Oniscidea). Misc. Zool. Hung. 12, 21-44.

[51] Schmalfuss, H. (ed.). (2003), World catalog of terrestrial isopods (Isopoda: Oniscidea). Stuttgarter Beitr. Naturk. Ser. A. Nr. 654.

[52] Korsós, Z., Hornung, E., Szlávecz, K., Kontschán, J. (2002), Isopoda and Diplopoda of urban habitats: new data to the fauna of Budapest. Ann. Zool. Nat. Hist. Mus. Hung. 94, 193-208.

[53] Sabino-Marques, H., Mira, A. (2011), Living on the verge: are roads a more suitable refuge for small mammals than streams in Mediterranean pastureland? Ecol. Res. 26(2), 277-287.

[54] Podlussány, A., Szita, É., Lupták, R., Szénási, V., Kiss, B. (2014), Four weevil species new to the fauna of Hungary from motorway rest areas (Coleoptera: Curculionidae). Fol. Entomol. Hungarica 75, 73-78. 